\title{
Predictive Value of the Pneumonia Severity Score on Mortality due to Aspiration Pneumonia
}

\author{
Nobutaka Hirooka, MD, MS, PhD, FAAFP; Tomohiro Nakayama, MD; \\ Takehito Kobayashi, MD, PhD; and Hidetomo Nakamoto, MD, PhD
}

\begin{abstract}
Objective: Designing an efficient management strategy for aspiration is of high priority in our aging society because of its high incidence. We evaluated the prognostic value of both the A-DROP (age, dehydration, respiratory, disorientation, and pressure) and the modified A-DROP scoring systems (adding respiratory rate and comorbidity to A-DROP) in patients with aspiration pneumonia.
\end{abstract}

Design: This is a retrospective study using electronic medical records at Saitama Medical University (SMU) hospital.

Setting: A 965-bed university tertiary medical center in Japan.

Participants: Data were extracted from the electronic medical records of patients from SMU hospital.

Methods: In-hospital mortality was compared between two groups: (I) those with a 'severe' to 'advanced severe' A-DROP score; and (2) those with a 'low' to 'middle' A-DROP score. Area under the curve (AUC) for mortality for both the A-DROP and modified A-DROP scoring systems were compared.

Results: The in-hospital mortality rates for patients with a high and a low A-DROP score were $28.6 \%$ and $9.0 \%$, respectively. The mortality rates in the high modified A-DROP score group and in the low modified A-DROP score group were $28.2 \%$ and $9.9 \%$, respectively. These differences in the mortality rates between the two groups were statistically significant for both the A-DROP and the modified A-DROP scoring systems. The AUC of the receiver operating characteristics curve for the A-DROP (0.700; $95 \%$ confidence interval, $0.608-0.779)$ was statistically significant.

Conclusion: The A-DROP and modified A-DROP scoring systems are associated with in-hospital mortality in patients with aspiration pneumonia. The A-DROP scoring system is easy to use and may be a clinically valuable tool in the management of aspiration pneumonia.

Keywords: Aspiration pneumonia; A-DROP; Modified A-DROP; Mortality; Prognosis

Corresponding Author: Nobutaka Hirooka MD, MS, PhD, Department of General Internal Medicine, Saitama Medical University, Morohongo 38, Moroyama-chou, Iruma-gun, Saitama, Japan 350-0495, E-mail: nkaorohi@saitama-med.ac.jp
Received: February 17,2020

Revised: November 16, 2020

Accepted: December 9, 2020

doi: $10.3121 / \mathrm{cmr} .2020 .1560$ 
$\mathrm{P}$ neumonia is a common disease and is a major cause of death. In the elderly population, in particular, it is a leading cause of death..$^{1-3}$ Thus, in a rapidly aging society such as that of Japan, pneumonia is of major clinical concern.

Aspiration pneumonia accounts for $5 \%$ to $24 \%$ of pneumonia cases. $^{4-7}$ Aspiration pneumonia is an infection of the lung parenchyma caused by inhaling gastric or oropharyngeal content into the lower respiratory tract. ${ }^{8}$ It primarily affects older adults, with $80 \%$ of those affected being 65 years-of-age or older. ${ }^{9}$ Previous studies have shown that aspiration pneumonia is associated with a higher mortality rate and longer hospital stays compared to other causes of pneumonia. ${ }^{10-12}$ Therefore, an efficient management strategy for the treatment of aspiration pneumonia is of high clinical priority in this aging society.

It is important to assess disease severity to plan and stratify the care of pneumonia patients, because identifying patients likely to have a major adverse outcome is a first step in reducing mortality rates. ${ }^{13}$ Assessing severity also guides therapeutic options such as the need for hospital or intensive care admission, suitability for discharge home, the extent of investigation, and the choice and route of administration of an antimicrobial agent. ${ }^{14-16}$ Several severity scoring systems such as the Pneumonia Severity Index (PSI) and the Confusion, Urea, Respiratory rate, Blood pressure plus age $\geq 65$ years (CURB-65) are available for the stratification of pneumonia cases, and these scoring systems not only denote disease severity but have also been shown to be associated with prognosis. ${ }^{16-19}$ Thus, it is useful for clinicians to use a severity scoring system in deciding upon a treatment strategy, including the assignment of the appropriate care setting, ie, outpatient, hospital, or intensive care.

In Japan, the A-DROP (age, dehydration, respiratory, disorientation, and pressure) scoring system has been adopted by the Japanese Respiratory Society (JRS).$^{20}$ The A-DROP was adapted from the CURB-65 by taking oxygen saturation into account in the scoring system instead of respiration rate. There have been reports that, similar to other pneumonia severity scoring systems, the A-DROP scoring system is associated with disease prognosis. ${ }^{21-23}$ Thus, the A-DROP is frequently used in the clinical setting to assess severity and is also used for the management of pneumonia in Japan. A modified A-DROP score, which adds the respiration rate and data on comorbidities to the A-DROP score, was reported to be better for the prediction of prognosis in pneumonia. Tateyama et $\mathrm{al}^{24}$ reported that adding 1 point to the A-DROP score if respiratory rate is 30 breaths per minute or more, or if the patient has comorbidities including congestive heart failure, cerebrovascular disease, renal disease, chronic obstructive pulmonary disease, or any type of cancer, increased the area under the curve (AUC) of receiver operating characteristics (ROC) from 0.807 to 0.840 .

While pneumonia severity scoring systems are widely used in the clinical setting to stratify pneumonia management efficiently, some studies have reported that severity scoring systems do not predict prognosis accurately in the case of aspiration pneumonia. ${ }^{25,26}$ Additionally, most studies on the use of scoring systems for ascertaining the prognosis of pneumonia have excluded cases of aspiration pneumonia. ${ }^{13,16}$ At present, further evidence is needed to elucidate whether severity scoring systems such as the A-DROP or the modified A-DROP scores can predict prognosis for aspiration pneumonia. In this study, we evaluated the prognostic value of both the A-DROP and the modified A-DROP scoring systems in the setting of aspiration pneumonia.

\section{Methods}

\section{Study Design and Setting}

This was a retrospective study using the electronic medical records (EMRs) of Saitama Medical University (SMU) hospital in Japan for the study period from January 1, 2017 to April 16, 2018. SMU hospital has 965 beds and plays an important role in providing care as a tertiary medical center. In this retrospective analysis of data from EMRs, cases of inpatient aspiration pneumonia were identified by using the International Classification of Diseases, $10^{\text {th }}$ edition (ICD-10) code specific to aspiration pneumonia and pneumonitis (J690).

Sample size calculation was performed before conducting the study. Based on a previously reported study conducted in Japan, ${ }^{27}$ the 30-day mortality of aspiration pneumonia in the low to middle and severe categories of the A-DROP score were $2.5 \%$ and $19.4 \%$, respectively. We used study type I error $(\alpha)=$ 0.05 and type II error $(\beta)=0.20$ in the sample size calculation. ${ }^{28}$ The calculation indicated that 52 cases in each group would constitute an appropriate sample size. Thus, 120 cases in total were chosen as the total sample size in this study, to account for a margin of error.

The enrolled cases were independently reviewed by two investigators (N.H. and T.N.). After case identification and review, two investigators reached consensus that all but one of the identified cases (age less than 20 years old at the time of hospitalization) met the inclusion criteria (see below). The study was approved by SMU hospital institutional review board (\#18161), and we obtained IRB agreement for waiver of patients' consent before embarking on retrospective record review study.

\section{Inclusion and Exclusion Criteria}

The criteria for inclusion were: (1) age $\geq 20$ years; (2) inpatient; (3) ICD-10 code indicating aspiration pneumonia as the primary diagnosis (J690). To exclude patients with recurrent pneumonia episodes, only the first hospitalization with aspiration pneumonia was chosen in the data analysis.

\section{Measurements}

The independent variable was the A-DROP score. To determine the A-DROP score, we extracted the first measurement of vital signs on admission, including oxygen saturation $\left(\mathrm{SpO}_{2}\right)$, age, mental status, and the first laboratory data from the EMRs, and 
then calculated the A-DROP score for each case based on the JRS guideline. An A-DROP score (consisting of age $\geq 70$ years in males or $\geq 75$ years in females, blood urea nitrogen $\geq$ $21 \mathrm{mg} / \mathrm{dL}$ or signs of dehydration, oxygen saturation measured by pulse oximetry $\leq 90 \%$, confusion, and systolic blood pressure $\leq 90$ $\mathrm{mmHg}$ ) was calculated by giving one point for each feature if present (range 0-5). When $\mathrm{SpO}_{2}$ was not available, data from arterial blood gas analysis (partial pressure of oxygen $\left[\mathrm{PaO}_{2}\right] \leq$ 60 Torr) was alternatively used to determine oxygen saturation. Data on comorbidities (congestive heart failure, cerebrovascular disease, renal disease, chronic obstructive pulmonary disease, and any type of cancer) for the calculation of the modified A-DROP scores were extracted from the EMRs. Residence before admission and performance status (PS) were also extracted from EMRs to further describe the basic characteristics of the study participants. The primary outcome was in-hospital mortality. We also analyzed 30-day mortality. Based on the collected data, we calculated the area under the receiver operating characteristics curve (AUC) for both the A-DROP and the modified A-DROP scoring systems in relation to in-hospital mortality.

\section{Statistical Analysis}

Descriptive analysis was performed using numbers, medians, and interquartile ranges (IQRs) for numeric values. For categorical data, counts and proportions were calculated as percentages. We compared the in-hospital mortality and 30-day mortality between the severe to advanced severe group (A-DROP score 3 to 5) and the low to middle group (A-DROP score 0 to 2). Similarly, in-hospital mortality was compared between those that had high (score between 4 to 7) versus low (score between 0 to 3 ) scores on the modified A-DROP scoring system. The chi-square test was used to determine the difference in mortality between the two groups. AUC was calculated both for the A-DROP score and the modified A-DROP score. Then, AUCs were compared statistically. ${ }^{29}$ A two-tailed $P$ value of $<0.05$ was considered to be statistically significant. IBM SPSS Statistics (Version 26.0. Armonk, NY) was used for the analysis.

\section{Results}

In total, 121 cases of aspiration pneumonia with hospitalization during the study period were identified retrospectively. One case was excluded, and the final sample size was 120 . Table 1 shows the study participants' characteristics. There were 81 males and 39 females enrolled in this study. The median ages (IQRs) of the overall, male, and female participants were 78.0 (19.0), 76.0 (21.0), and 82.0 (17.0) years, respectively. In the A-DROP scores, altered mental status was the most common factor found among the participants, followed by dehydration, hypoxia, and hypotension (Table 1). Comorbidities including cerebrovascular disease, renal disease, congestive

Table 1. Demographic characteristics of study participants

\begin{tabular}{|c|c|c|c|}
\hline & Total & Male & Female \\
\hline Sex, n (\%) & $120(100)$ & $81(67.5)$ & 39 (32.5) \\
\hline \multicolumn{4}{|l|}{ Age, n (\%) } \\
\hline$<40$ & $6(5.0)$ & $5(6.2)$ & $1(2.6)$ \\
\hline $41-50$ & $4(3.3)$ & $3(3.7)$ & $1(2.6)$ \\
\hline $51-60$ & $9(7.5)$ & $8(9.9)$ & $1(2.6)$ \\
\hline $61-70$ & $17(14.2)$ & $12(14.8)$ & $5(12.8)$ \\
\hline $71-80$ & $34(28.3)$ & $28(34.6)$ & $6(15.4)$ \\
\hline $81-90$ & $37(30.8)$ & $22(27.2)$ & $15(38.5)$ \\
\hline$>91$ & $13(10.8)$ & $3(3.7)$ & $10(25.6)$ \\
\hline Median age, years (IQR) & $78.0(19.0)$ & $76.0(21.0)$ & $82.0(17.0)$ \\
\hline \multicolumn{4}{|l|}{ Residence n (\%) } \\
\hline Home & $60(50.0)$ & $40(49.3)$ & $20(51.3)$ \\
\hline Nursing home & $44(36.7)$ & $29(35.8)$ & $15(38.5)$ \\
\hline Hospital & $16(13.3)$ & $12(14.8)$ & $4(10.3)$ \\
\hline \multicolumn{4}{|l|}{ Performance Status n (\%) } \\
\hline 0 & $3(2.5)$ & $2(3.3)$ & $1(2.6)$ \\
\hline 1 & $7(5.8)$ & $4(6.6)$ & $3(7.7)$ \\
\hline 2 & $29(24.2)$ & $23(37.7)$ & $6(15.4)$ \\
\hline 3 & $18(15.0)$ & $7(11.5)$ & $11(28.2)$ \\
\hline 4 & $61(50.8)$ & $43(70.5)$ & $18(46.2)$ \\
\hline Unknown & $2(1.7)$ & $2(3.3)$ & $0(0)$ \\
\hline \multicolumn{4}{|l|}{ A-DROP, n (\%) } \\
\hline \multicolumn{4}{|l|}{ Age } \\
\hline Male $\geq 70 \mathrm{yr}$ & $55(45.8)$ & - & - \\
\hline Female $\geq 75 \mathrm{yr}$ & $30(25.0)$ & - & - \\
\hline Altered mental status & $72(60.0)$ & $46(51.7)$ & $26(66.7)$ \\
\hline Systolic blood pressure $\leq 90 \mathrm{mmHg}$ & $7(5.8)$ & $4(4.5)$ & $3(7.7)$ \\
\hline Dehydration & $54(45.0)$ & $36(40.4)$ & $18(46.2)$ \\
\hline $\mathrm{SpO}_{2} \leq 90 \%$ or $\mathrm{PaO}_{2} \leq 60$ Torr & $33(27.5)$ & $25(28.1)$ & $8(20.5)$ \\
\hline \multicolumn{4}{|l|}{ Additional risk, $\mathrm{n}(\%)$} \\
\hline Respiratory rate & $20(16.7)$ & $15(16.9)$ & $5(12.8)$ \\
\hline \multicolumn{4}{|l|}{ Co-morbidity } \\
\hline Liver disease & $9(7.5)$ & $7(7.9)$ & $1(2.6)$ \\
\hline Cerebrovascular disease & $49(40.8)$ & $37(41.6)$ & $12(30.8)$ \\
\hline Congestive heart failure & $18(15.0)$ & $11(12.4)$ & $7(17.9)$ \\
\hline Neoplastic disease & $17(14.2)$ & $15(16.9)$ & $2(5.1)$ \\
\hline COPD & $6(5.0)$ & $5(5.6)$ & $1(2.6)$ \\
\hline Renal disease & $20(16.7)$ & $15(16.9)$ & $5(12.8)$ \\
\hline
\end{tabular}


Table 2. Distribution of A-DROP score and in-hospital mortality $(\mathrm{N}=120)$

\begin{tabular}{rccc}
\hline Score & Number of patients, $\mathbf{n}(\%)$ & In-hospital mortality, $\mathbf{n}(\%)$ & Group mortality, $\mathbf{n}(\%)$ \\
\hline 0 & $3(2.5)$ & $1(33.3)$ & \\
1 & $35(29.2)$ & $2(5.7)$ & Low 7 (9.0) \\
2 & $40(33.3)$ & $4(10.0)$ & \\
\hline 3 & $34(28.3)$ & $7(20.6)$ & High $12(28.6)$ \\
4 & $7(5.8)$ & $4(57.1)$ & \\
5 & $1(0.8)$ & $1(100.0)$ &
\end{tabular}

heart failure, and neoplastic disease were relatively prevalent among the study participants. There were only nine cases of liver disease and six cases of COPD among study participants, as shown in Table 1.

There were 19 in-hospital deaths in the study population for median follow-up period of 27.0 days. Table 2 shows the number of deaths for each A-DROP score stratification. The rates of in-hospital death were 1/3 (33.3\%), 2/35 (5.7\%), 4/40 (10.0\%), 7/34 (20.6\%), 4/7 (57.1\%), and 1/1 (100.0\%) for A-DROP scores of $0,1,2,3,4$, and 5 , respectively. The in-hospital mortality rate was $28.6 \%$ in the high score group (A-DROP score $\geq 3$ ) and was $9.0 \%$ in the low score group $($ A-DROP $<3)$. There was a statistically significant difference in the in-hospital mortality rate between the two groups $(P$ $=0.005$ ). In the 30-day mortality analysis, there was also a statistically significant difference between the low and high A-DROP score groups $(P<0.01)$. The 30 -day mortality was $9.6 \%$ (7 deaths out of 73 patients) and 30.0\% (12 deaths out of 40 patients) for low score and high score group, respectively.

Table 3 shows the in-hospital mortality rates by the modified A-DROP scoring system. Of the 19 deaths that occurred in our study population, the rates of death for each modified A-DROP score were 0/0 (0\%), 2/7 (28.6\%), 2/37 (5.4\%), 5/38 (13.2\%), 5/25 (20.0\%), 2/9 (22.2\%), 2/3 (66.7\%) and 1/1 $(100.0 \%)$ for scores of $0,1,2,3,4,5,6$ and 7 , respectively. The mortality rate was $28.2 \%$ in the high score group (modified A-DROP score $\geq 4$ ) and was $9.9 \%$ in the low score group (modified A-DROP $<4$ ). The difference in mortality rate between the two groups was statistically significant $(P$ $=0.03$ ). In the 30-day mortality analysis, there was also a statistically significant difference between the low and high modified A-DROP score groups $(P<0.05)$. The 30-day mortality was $11.7 \%$ ( 9 deaths out of 77 patients) and $28.6 \%$ (10 deaths out of 35 patients) for low score and high score group, respectively.

The AUC of the A-DROP scoring system was 0.700 , with a $95 \%$ confidence interval of $0.608-0.779$, and this was statistically significant from random classifier $(P=0.0064)$. For the modified A-DROP scoring system, the AUC was $0.647(P=0.0563)$, with a $95 \%$ confidence interval of 0.555 0.732. Based on the ROC data, the AUCs were compared between the A-DROP and modified A-DROP scoring systems, and there was no statistically significant difference between the AUCs of the two scoring systems $(P=0.091)$.

\section{Discussion}

The present study found that the A-DROP and the modified A-DROP scoring systems predicted the prognosis of aspiration pneumonia in a statistically significant manner. However, the modified A-DROP scoring system, which counts respiration rate and comorbid disease into the score of the original A-DROP system, did not add prognostic value to the A-DROP scoring system in these patients. These results suggested the AUC of the A-DROP scoring system is an acceptable tool for predicting prognosis in aspiration pneumonia. ${ }^{30}$

Our study findings indicate the inpatient mortality rate of aspiration pneumonia was $15.8 \%$. This is in line with other studies. ${ }^{27,31-33}$ The mortality of aspiration pneumonia is higher than the mortality rate of non-aspiration pneumonia. Mortality rates ranging from $12.9 \%$ to $59.1 \%$ have been reported for aspiration pneumonia (average $17.8 \%$ ), and a range of $2.5 \%$ to $22.8 \%$ has been reported for non-aspiration pneumonia

Table 3. Distribution of modified A-DROP score and in-hospital mortality $(\mathrm{N}=120)$

\begin{tabular}{cccc}
\hline Score & Number of patients, $\mathbf{n}(\%)$ & In-hospital mortality, $\mathbf{n}(\%)$ & Group mortality, $\mathbf{n}(\%)$ \\
\hline 0 & $0(.0)$ & $0(.0)$ & \\
1 & $7(5.8)$ & $2(28.6)$ & Low $9(9.9)$ \\
2 & $37(30.8)$ & $2(5.4)$ & \\
3 & $38(31.7)$ & $5(13.2)$ & \\
\hline 4 & $25(20.8)$ & $5(20.0)$ & High $10(28.2)$ \\
5 & $9(7.5)$ & $2(22.2)$ & \\
6 & $3(2.5)$ & $2(66.7)$ & \\
7 & $1(0.8)$ & $1(100.0)$ & \\
\hline
\end{tabular}


(average 5.9\%). ${ }^{27}$ It has previously been reported that aspiration pneumonia accounts for between $5 \%$ and $24 \%$ of community acquired pneumonia. ${ }^{27,33}$ Because of the higher mortality associated with it, aspiration pneumonia requires precise therapy strategies to avoid a negative outcome.

In this study, the A-DROP scoring system was shown to be useful for predicting prognosis in aspiration pneumonia. The AUC of the A-DROP scoring system for the prediction of mortality in aspiration pneumonia was 0.700 , which is a statistically significant outcome. The value of the AUC is considered clinically acceptable as a diagnostic test. ${ }^{30}$ Therefore, the A-DROP scoring system can be a useful tool for guiding therapeutic options in clinical practice. Previous studies have suggested that pneumonia severity scoring systems may better predict mortality in community acquired pneumonia (CAP) than in aspiration pneumonia, ${ }^{25}$ while the AUC of the A-DROP scoring system in the present study is slightly higher than the same value in previous studies..$^{25,34}$ Recently, Ito et $\mathrm{al}^{35}$ reported on comparative evaluation of pneumonia severity scoring systems in nursing and healthcareassociated pneumonia (NHCAP) in which cases of aspiration pneumonia are included. It showed there were differences among the type of severity scoring systems in predicting mortality among the Japanese patients. While the study participants were different, the AUC of PSI was the highest (0.717; 95\% confidence interval [0.673-0.761]) as compared to other severity scores including A-DROP. However, the AUC is comparable with our results using A-DROP. The difference may be associated with the difference in study participants, such as type of pneumonia. Further investigation to improve the ability of scoring systems to predict mortality in aspiration pneumonia is of further research interest.

Comparing AUCs between the A-DROP and the modified A-DROP scores sheds important light on the characteristics of aspiration pneumonia. Based on the results of our study, aspiration pneumonia may be a distinct entity from other types of pneumonia, in that comorbidities did not add to the predictive value of the modified A-DROP score. Pathophysiologically, aspiration pneumonia is different from non-aspiration pneumonia, because aspiration pneumonia not only involves the infiltration of bacterial pathogens, but also comprises inflammation due to the inhalation of gastric or oropharyngeal contents. ${ }^{34}$ As discussed previously, many reports have shown aspiration pneumonia carries a higher mortality than nonaspiration pneumonia. ${ }^{27}$ Several studies have shown prognostic prediction is more accurate when comorbidities and disease severity are taken into account for CAP. Tateyama et $\mathrm{al}^{24}$ reported that the inclusion of comorbidity and respiratory rate adds value to the A-DROP scoring system for Japanese CAP patients. Higuchi et $\mathrm{al}^{21}$ also studied the prediction of mortality among elderly Japanese patients, using the same scoring system and reporting on the same ethnic group as that of the present study, and concluded that the modified A-DROP scoring system increased the ability to predict mortality in
CAP. Conversely, several studies have concluded that the incorporation of data on comorbidities into existing pneumonia severity scores such as the PSI, Curb-65, and A-DROP may not increase the ability to predict mortality in the case of aspiration pneumonia. ${ }^{27,34}$ While this difference in the prediction of mortality between aspiration pneumonia and other pneumonia might be influenced by the background of the patients enrolled in each study, the disease entity of aspiration pneumonia is potentially the independent factor differentiating these scores from those relating to other causes of pneumonia. Taylor et $\mathrm{al}^{33}$ have stated a clinical phenotype for pneumonia. Aspiration pneumonia is an intuitive clinical subcategory of pneumonia. As discussed previously, the difference in mortality between non-aspiration pneumonia and aspiration pneumonia may be explained in terms of their pathophysiologies and risk factors. Given these differences between aspiration and non-aspiration pneumonia, aspiration pneumonia may be its own clinical phenotype. Ahn et $\mathrm{al}^{36}$ studied prediction models specific to the prediction of mortality in cancer patients with pneumonia and concluded that a new model using PS, $\mathrm{SpO}_{2}$, and lactic acidosis would be better for predicting mortality in these patients. Whether comorbidity-specific models yield better prediction outcomes in other phenotypes, including aspiration pneumonia, needs to be investigated further.

This study adds to the existing evidence for the value of the A-DROP scoring system in predicting the prognosis of aspiration pneumonia. Additionally, the study infers the concept that aspiration pneumonia is a disease entity that is distinct from other causes of pneumonia.

This study has several limitations. This is a retrospective study, which does not incorporate all possible confounders into its analysis. However, our results indicate that the modified A-DROP system, which incorporates factors known to better predict outcomes in CAP, confers no additional prognostic value over the A-DROP scoring system in the setting of aspiration pneumonia. These known factors were included for analysis in this study. Other factors may need to be studied in the future. In keeping with other studies on aspiration pneumonia, our study used clinical determinants to define aspiration pneumonia. Unfortunately, there is no standard definition for aspiration pneumonia. Additionally, elderly patients are more likely than younger patients to be diagnosed with aspiration pneumonia, resulting in an agerelated bias in this study. However, our institution uses the definition of aspiration pneumonia by the JRS, in which aspiration pneumonia is defined as pneumonia that develops in patients in whom dysphagia and aspiration is known to occur (or is strongly suspected). It is diagnosed by clinical findings, image studies, and findings such as evidence of aspiration, dysphasia, or underlying conditions for abnormal swallowing function. ${ }^{37}$ Each of the ICD10 codes for patients was reviewed and finalized by the physicians in charge of the cases in the EMRs at the time of discharge. Then, the finalized 
ICD10 codes were used in this study. Thus, a majority of the aspiration pneumonia cases were uniformly diagnosed based on the JRS criteria. Future studies on aspiration pneumonia should assure the case selection by the established criteria and the process of clinical diagnosis of aspiration pneumonia to decrease bias.

\section{Conclusion}

A widely used pneumonia severity scoring system, the A-DROP score, was shown to be associated with prognosis in cases of aspiration pneumonia. The modified A-DROP did not add prognostic value over the original A-DROP score in the setting of aspiration pneumonia. No additional prognostic value was added by the incorporation of comorbidities into the score. Thus, our study inferred the concept that aspiration pneumonia is a distinct disease entity and is different from other causes of pneumonia. The A-DROP scoring system maybe a valuable tool in the clinical management of aspiration pneumonia.

\section{Acknowledgements}

We would like to thank Editage (www.editage.com) for English language editing.

\section{References}

1. Miyashita N, Yamauchi Y. Bacterial pneumonia in elderly Japanese populations. Jpn Clin Med. 2018;9:1179670717751433.

2. Zheng Y, Chang Q, Yip PSF. Understanding the increase in life expectancy in Hong Kong: contributions of changes in age- and cause-specific mortality. Int J Environ Res Public Health. 2019;16(11):1959.

3. Yoon HK. Changes in the epidemiology and burden of community-acquired pneumonia in Korea. Korean J Intern Med. 2014;29(6):735-737.

4. Reza Shariatzadeh M, Huang JQ, Marrie TJ. Differences in the features of aspiration pneumonia according to site of acquisition: community or continuing care facility. $\mathrm{J}$ Am Geriatr Soc. 2006;54(2):296-302.

5. Moine P, Vercken JB, Chevret S, Chastang C, Gajdos P. Severe community-acquired pneumonia. Etiology, epidemiology, and prognosis factors. French Study Group for Community-Acquired Pneumonia in the Intensive Care Unit. Chest. 1994;105(5):1487-1495.

6. Torres A, Serra-Batlles J, Ferrer A, et al. Severe community-acquired pneumonia. Epidemiology and prognostic factors. Am Rev Respir Dis. 1991;144(2): 312-318.

7. Marrie TJ, Durant H, Yates L. Community-acquired pneumonia requiring hospitalization: 5 -year prospective study. Clin Infect Dis. 1989;11(4):586-599.

8. Mandell LA, Niederman MS. Aspiration Pneumonia. N Engl J Med. 2019;380(7):651-663.
9. Wu CP, Chen YW, Wang MJ, Pinelis E. National trends in admission for aspiration pneumonia in the United States, 2002-2012. Ann Am Thorac Soc. 2017;14(6):874879.

10. Reza Shariatzadeh M, Huang JQ, Marrie TJ. Differences in the features of aspiration pneumonia according to site of acquisition: community or continuing care facility. J Am Geriatr Soc. 2006;54(2):296-302.

11. Hayashi M, Iwasaki T, Yamazaki Y, et al. Clinical features and outcomes of aspiration pneumonia compared with non-aspiration pneumonia: A retrospective cohort study. J Infect Chemother. 2014;20(7):436-442.

12. Jeon I, Jung GP, Seo HG, Ryu JS, Han TR, Oh BM. Proportion of aspiration pneumonia cases among patients with community-acquired pneumonia: a single-center study in Korea. Ann Rehabil Med. 2019;43(2):121-128.

13. España PP, Capelastegui A, Gorordo I, et al. Development and validation of a clinical prediction rule for severe community-acquired pneumonia. Am J Respir Crit Care Med. 2006;174(11):1249-1256.

14. Bartlett JG, Dowell SF, Mandell LA, File TM Jr, Musher DM, Fine MJ. Practice guidelines for the management of community-acquired pneumonia in adults. Infectious Diseases Society of America. Clin Infect Dis. 2000;31(2):347-382.

15. Mandell LA, Marrie TJ, Grossman RF, Chow AW, Hyland RH. Canadian guidelines for the initial management of community-acquired pneumonia: an evidence-based update by the Canadian Infectious Diseases Society and the Canadian Thoracic Society. The Canadian Community-Acquired Pneumonia Working Group. Clin Infect Dis. 2000;31(2):383-421.

16. Lim WS, van der Eerden MM, Laing R, et al. Defining community acquired pneumonia severity on presentation to hospital: an international derivation and validation study. Thorax. 2003;58(5):377-382.

17. Fine MJ, Auble TE, Yealy DM, et al. A prediction rule to identify low-risk patients with community-acquired pneumonia. N Engl J Med. 1997;336(4):243-250.

18. Mandell LA, Wunderink RG, Anzueto A, et al. Infectious Diseases Society of America/American Thoracic Society consensus guidelines on the management of community-acquired pneumonia in adults. Clin Infect Dis. 2007;44(Suppl 2):S27-S72.

19. Lim WS, Baudouin SV, George RC, et al. BTS guidelines for the management of community acquired pneumonia in adults: update 2009. Thorax. 2009;64 Suppl 3:iii1-iii55.

20. Miyashita N, Matsushima T, Oka M, Japanese Respiratory Society. The JRS guidelines for the management of community-acquired pneumonia in adults: an update and new recommendations. Intern Med. 2006;45(7):419-428. 
21. Higuchi T, Ota K, Tanabe Y, Suzuki E, Gejyo F. Nihon Ronen Igakkai Zasshi. 2007;44(4):483-489.

22. Ahn JH, Choi EY. Expanded A-DROP Score: A new scoring system for the prediction of mortality in hospitalized patients with community-acquired pneumonia. Sci Rep. 2018;8(1):14588.

23. Shindo Y, Sato S, Maruyama E, et al. Comparison of severity scoring systems A-DROP and CURB-65 for community-acquired pneumonia. Respirology. 2008;13(5):731-735.

24. Tateyama M, Shinzato T, Haranaga S, et al. Nihon Kokyuki Gakkai Zasshi. 2011;49(5):343-348.

25. Lanspa MJ, Jones BE, Brown SM, Dean NC. Mortality, morbidity, and disease severity of patients with aspiration pneumonia. J Hosp Med. 2013;8(2):83-90.

26. Heppner HJ, Sehlhoff B, Niklaus D, Pientka L, Thiem U. Pneumonie-Schwere-Index (PSI), CURB-65 und Mortalität bei hospitalisierten geriatrischen Patienten mit Aspirationspneumonie [Pneumonia Severity Index (PSI), CURB-65, and mortality in hospitalized elderly patients with aspiration pneumonia]. Z Gerontol Geriatr. 2011;44(4):229-234.

27. Komiya K, Rubin BK, Kadota JI, et al. Prognostic implications of aspiration pneumonia in patients with community acquired pneumonia: A systematic review with meta-analysis. Sci Rep. 2016;6:38097.

28. Columb MO, Atkinson MS. Statistical analysis: sample size and power estimations. BJA Educ. 2016;16(5): 159-161.

29. DeLong ER, DeLong DM, Clarke-Pearson DL. Comparing the areas under two or more correlated receiver operating characteristic curves: a nonparametric approach. Biometrics. 1988;44(3):837-845.

30. Mandrekar JN. Receiver operating characteristic curve in diagnostic test assessment. J Thorac Oncol. 2010;5(9):1315-1316.

31. Nakagawa N, Saito Y, Sasaki M, Tsuda Y, Mochizuki $\mathrm{H}$, Takahashi H. Comparison of clinical profile in elderly patients with nursing and healthcare-associated pneumonia, and those with community-acquired pneumonia. Geriatr Gerontol Int. 2014;14(2):362-371.

32. Hsu JL, Siroka AM, Smith MW, Holodniy M, Meduri GU. One-year outcomes of communityacquired and healthcare-associated pneumonia in the Veterans Affairs Healthcare System. Int J Infect Dis. 2011;15(6):e382-e387.

33. Taylor JK, Fleming GB, Singanayagam A, Hill AT, Chalmers JD. Risk factors for aspiration in communityacquired pneumonia: analysis of a hospitalized UK cohort. Am J Med. 2013;126(11):995-1001.
34. Lanspa MJ, Peyrani P, Wiemken T, Wilson EL, Ramirez JA, Dean NC. Characteristics associated with clinician diagnosis of aspiration pneumonia: A descriptive study of afflicted patients and their outcomes. J Hosp Med. 2015;10(2):90-96.

35. Ito A, Ishida T, Tokumasu H, Yamazaki A, Washio Y. Evaluation of pneumonia severity scoring systems in nursing and healthcare-associated pneumonia for predicting prognosis: A prospective, cohort study. J Infect Chemother. 2020;26(4):372-378.

36. Ahn BK, Lee YS, Kim YJ, et al. Prediction model for mortality in cancer patients with pneumonia: comparison with CURB-65 and PSI. Clin Respir J. 2018;12(2):538546.

37. Kohno S, Imamura Y, Shindo Y, et al. Clinical practice guidelines for nursing- and healthcare-associated pneumonia (NHCAP) [complete translation]. Respir Investig. 2013;51(2):103-126.

\section{Author Affiliations}

Nobutaka Hirooka, MD, MS, PhD, FAAFP*; Tomohiro

Nakayama, MD*; Takehito Kobayashi, MD, PhD*; and

Hidetomo Nakamoto, $M D, P h D^{*}$

*Department of General Internal Medicine, Saitama Medical University, Morohongo 38, Moroyama-chou, Iruma-gun, Saitama, Japan 350-0495 